\title{
Convenient Synthesis of Aromaric Poly(ether ketone)s Containing Alicyclic Units
}

\author{
By Katsuya MaEYAma, ${ }^{*}$ Takeshi KAmURA, Keita AKIBA, and Hiromu SAITO
}

Aromatic poly(ether ketone)s containing cyclohexanediyl and adamantanediyl units were synthesized through nucleophilic aromatic substitution polymerization of alicyclic difluorides with bisphenols in the presence of $\mathrm{K}_{2} \mathrm{CO}_{3}$ in $\mathrm{NMP}$. Most of the poly(ether ketone)s thus synthesized are soluble in organic solvents such as $\mathrm{CHCl}_{3}$ and THF. Glass transition temperatures ( $T_{\mathrm{g}}$ 's) of the polymers and temperatures where $5 \%$ weight losses occur are in the region of $146-199{ }^{\circ} \mathrm{C}$ and $432-500{ }^{\circ} \mathrm{C}$, respectively, which shows that the poly(ether ketone)s have excellent thermal stability. UV spectra of the poly(ether ketone)s revealed that they are sufficiently transparent in the wavelength above $310 \mathrm{~nm}$.

KEY WORDS: Semi-aromatic Poly(ether ketone)s / Nucleophilic Aromatic Substitution Polymerization / Thermal Stability / Excellent Solubility /

Aromatic poly(ether ketone)s, which consists of aromatics, ketone groups, and ether units, have much attraction as physically and chemically stable organic materials., ${ }^{1,2}$ In particular, PEEK has been well-known as one of super engineering plastics. We have also synthesized aromatic poly(ether ketone) $\mathrm{s}^{3,4}$ and wholly aromatic polyketones ${ }^{5}$ containing optically active 2,2'-dimethoxy-1,1'-binaphthyl-6, $6^{\prime}$ diyl units. The resulting polymers have high thermal stability and excellent solubility in organic solvents such as $\mathrm{CHCl}_{3}$ and DMF. On the other hand, polyimides, ${ }^{6-8}$ poly(benzoxazole)s, ${ }^{9}$ and polyolefins ${ }^{10,11}$ incorporating alicyclic units derived from norbornene and adamantane derivatives have been recently much reported. Incorporation of alicyclic units to aromatic polymer skeletons increases transparency associating with small decrease of thermal stability. We have also synthesized semi-aromatic polyesters incorporating alicyclic units, i.e., cyclohexanediyl and adamantanediyl ones, in the main chains. ${ }^{12}$ The resulting polyesters have also high thermal stability $\left(T_{\mathrm{g}} 222^{\circ} \mathrm{C}\right)$ and excellent solubility in organic solvents such as $\mathrm{CHCl}_{3}$ and DMF as well as good transparency. These motivated us to develop poly(ether ketone)s consisting of both aromatic and alicyclic units, which should be applied to highperformance materials including optical materials.

In this paper, we report synthesis of poly(ether ketone)s through nucleophilic aromatic substitution polymerization of difluorides containing alicyclic units with bisphenols in the presence of $\mathrm{K}_{2} \mathrm{CO}_{3}$. Thermal and optical properties and solubility in organic solvents of the resulting poly(ether ketone)s are also described.

\section{EXPERIMENTAL}

\section{Materials \\ Cyclohexanedicarboxylic acids, fluorobenzene, aromatic diols 5c,d, and $\mathrm{PCl}_{5}$ were purchased from Tokyo Kasei Kogyo}

Co. Ltd. Aromatic diols 5a,b were purchased from Wako Pure Chemical Industries. A THF solution of 4-fluorophenylmagnesium bromide was purchased from Aldrich. These reagents were used as received. DMF and NMP were purchased from Kanto Chemical Co. Ltd. and used after distillation under reduced pressure. Cyclohexanedicarbonyl dichlorides $\mathbf{1}$ and $\mathbf{3}$ were prepared through the reaction of the corresponding cyclohexanedicarboxylic acid with $\mathrm{PCl}_{5}$ at room temperature. ${ }^{12}$ On the other hand, 1,3-adamantanedicarbonyl dichloride (8) was prepared through the reaction of 1,3-adamantanedicarboxylic acid with $\mathrm{SOCl}_{2}$.

\section{Instruments}

${ }^{1} \mathrm{H}$ NMR spectra were recorded on a JEOL JNM-AL-300 $(300 \mathrm{MHz})$. Chemical shifts were expressed in ppm relative to internal standard of $\mathrm{Me}_{4} \mathrm{Si}(\delta, 0.00) .{ }^{13} \mathrm{C}$ NMR spectra were recorded on a JEOL JNM-AL-300 (75 MHz). Chemical shifts were expressed in ppm relative to internal standard of $\mathrm{CDCl}_{3}$ $(\delta, 77.0)$. IR measurement was recorded on a JEOL FT/IR4100. Gel permeation chromatography (GPC) measurements were carried out at a flow rate of $1.0 \mathrm{~mL} \mathrm{~min}^{-1}$ at $40{ }^{\circ} \mathrm{C}$ using $\mathrm{CHCl}_{3}$ as an eluent on a Shimadzu SPD-6A equipped with a UV detector $(300 \mathrm{~nm})$ and a Shodex KD-806M column. Glass transition temperatures $\left(T_{\mathrm{g}}\right)$ were obtained on the basis of DSC thermograms. DSC thermograms were recorded on a Rigaku DSC-8230 differential scanning calorimeter with a heating rate of $10 \mathrm{~K} \mathrm{~min}^{-1}$. Thermal degradation temperatures $\left(T_{\mathrm{d} 5}\right)$ were obtained on the basis of TGA thermograms. The TGA thermograms were recorded on a Rigaku TG-8120 thermogravimeter with a heating rate of $10 \mathrm{~K} \mathrm{~min}^{-1}$. UV spectra were recorded on a JEOL V-630 spectrophotometer.

trans-1,2-Bis(4-fluorobenzoyl)cyclohexane (2)

To a dried $30 \mathrm{~mL}$ two-necked flask were added trans-1,2-

\footnotetext{
Department of Organic and Polymer Materials Chemistry, Graduate School of Technology, Tokyo University of Agriculture and Technology, Koganei 184-8588, Japan

*To whom correspondence should be addressed (Tel: +81-42-388-7475, Fax: +81-42-388-7291, E-mail: maeyama@cc.tuat.ac.jp).
} 
cyclohexanedicarbonyl dichloride $(\mathbf{1}, 212 \mathrm{mg}, 2 \mathrm{mmol})$, aluminium chloride (668 mg, $5 \mathrm{mmol}$ ), and 1,2-dichloroethane $(6 \mathrm{~mL})$. After the mixture was stirred for several minutes at $0{ }^{\circ} \mathrm{C}$, fluorobenzene $(1.15 \mathrm{~g}, 12 \mathrm{mmol})$ was added. The reaction mixture was stirred for $24 \mathrm{~h}$ at room temperature. The reaction mixture was quenched by pouring it into saturated $\mathrm{NaHCO}_{3}$ aq. in a beaker. The organic layer was extracted with chloroform three times. The combined extracts were dried over anhydrous $\mathrm{MgSO}_{4}$. After removal of the drying agent, chloroform was removed under reduced pressure. The crude product was purified by recrystalization from hexane $/ \mathrm{CHCl}_{3}$ to afford pure difluoride 2 in $65 \%$ yield.

Melting point $135-136{ }^{\circ} \mathrm{C} .{ }^{1} \mathrm{H} \mathrm{NMR} \delta\left(300 \mathrm{MHz}, \mathrm{CDCl}_{3}\right)$ : 1.31-1.59 (4H, m), 1.85-1.98 (2H, m), 2.02-2.15 (2H, m), 3.75-3.95 (2H, m), $7.13(4 \mathrm{H}, \mathrm{t}, J=9.0 \mathrm{~Hz}), 8.01(4 \mathrm{H}, \mathrm{dd}$, $J=5.4,9.0 \mathrm{~Hz}) \mathrm{ppm} .{ }^{13} \mathrm{C} \mathrm{NMR} \delta\left(75 \mathrm{MHz}, \mathrm{CDCl}_{3}\right): 25.9$, $30.1,47.1,115.7\left(\mathrm{~d},{ }^{2} J_{\mathrm{CF}}=22 \mathrm{~Hz}\right), 131.1\left(\mathrm{~d},{ }^{3} J_{\mathrm{CF}}=9 \mathrm{~Hz}\right)$, $132.5\left(\mathrm{~d},{ }^{4} J_{\mathrm{CF}}=3 \mathrm{~Hz}\right), 165.7\left(\mathrm{~d},{ }^{1} J_{\mathrm{CF}}=253 \mathrm{~Hz}\right), 201.8 \mathrm{ppm}$. IR $v$ (neat) $1673 \mathrm{~cm}^{-1}$. Anal. Calcd. for $\mathrm{C}_{20} \mathrm{H}_{18} \mathrm{~F}_{2} \mathrm{O}_{2}$ : C $73.16 \%, \mathrm{H} 5.53 \%$. Found, C 73.39\%, H 5.68\%.

\section{trans-1,4-Bis(4-fluorobenzoyl)cyclohexane (4)}

trans-1,4-Bis(4-fluorobenzoyl)cyclohexane (4) was prepared according to the synthesis of trans-1,2-bis(4-fluorobenzoyl)cyclohexane (2). Isolated yield. $78 \%$.

Melting point $208-213{ }^{\circ} \mathrm{C} .{ }^{1} \mathrm{H}$ NMR $\delta\left(300 \mathrm{MHz}, \mathrm{CDCl}_{3}\right)$ : 1.64-1.78 (4H, m), 1.95-2.19 (4H, m), 3.22-3.37 (2H, m), 7.16 $(4 \mathrm{H}, \mathrm{t}, J=8.4 \mathrm{~Hz}), 7.99(4 \mathrm{H}, \mathrm{dd}, J=5.1,8.4 \mathrm{~Hz}) \mathrm{ppm}$. ${ }^{13} \mathrm{C} \mathrm{NMR} \delta\left(75 \mathrm{MHz}, \mathrm{CDCl}_{3}\right): 28.6,44.7,115.7\left(\mathrm{~d},{ }^{2} J_{\mathrm{CF}}=\right.$ $26 \mathrm{~Hz}), 130.8\left(\mathrm{~d},{ }^{3} J_{\mathrm{CF}}=9 \mathrm{~Hz}\right), 132.3\left(\mathrm{~d},{ }^{4} J_{\mathrm{CF}}=3 \mathrm{~Hz}\right), 165.7$ $\left(\mathrm{d},{ }^{1} J_{\mathrm{CF}}=253 \mathrm{~Hz}\right), 201.8 \mathrm{ppm}$. IR $v($ neat $) 1679 \mathrm{~cm}^{-1}$. Anal. Calcd. for $\mathrm{C}_{20} \mathrm{H}_{18} \mathrm{~F}_{2} \mathrm{O}_{2}$ : C 73.16\%, H 5.53\%. Found, C 73.39\%, H $5.64 \%$.

\section{1,3-Bis(4-fluorobenzoyl)adamantane (9)}

To a dried $30 \mathrm{~mL}$ two-necked flask were added 1,3adamantanedicarbonyl dichloride $(\mathbf{8}, 391 \mathrm{mg}, 1.5 \mathrm{mmol})$, $\mathrm{CuCl}^{13}$ (10 mg, $\left.0.075 \mathrm{mmol}\right)$, and THF $(4 \mathrm{~mL})$. After the mixture was stirred for several minutes at $0{ }^{\circ} \mathrm{C}$, a $1 \mathrm{M} \mathrm{THF}$ solution of 4-fluorophenylmagnesium bromide $(3.5 \mathrm{~mL})$ was added dropwise. The reaction mixture was quenched by pouring it into $1 \mathrm{M} \mathrm{HCl}$ aq. in a beaker. The organic layer was extracted with diethyl ether three times. The combined extracts were dried over anhydrous $\mathrm{MgSO}_{4}$. After removal of the drying agent, diethyl ether was removed under reduced pressure. The crude product was purified by recrystalization from $\mathrm{CHCl}_{3} /$ hexane to afford pure difluoride 9 in $70 \%$ yield.

Melting point $118-120{ }^{\circ} \mathrm{C} .{ }^{1} \mathrm{H} \mathrm{NMR} \delta\left(300 \mathrm{MHz}, \mathrm{CDCl}_{3}\right)$ : 1.73-1.80 (2H, m), 1.90-2.15 (8H, m), 2.19-2.39 (4H, m), 7.09 $(4 \mathrm{H}, \mathrm{t}, J=8.7 \mathrm{~Hz}), 7.65(4 \mathrm{H}, \mathrm{dd}, J=5.1,8.7 \mathrm{~Hz}) \mathrm{ppm}$. ${ }^{13} \mathrm{C} \mathrm{NMR} \delta\left(75 \mathrm{MHz}, \mathrm{CDCl}_{3}\right): 28.1,35.5,38.3,40.3,47.2$, $115.2\left(\mathrm{~d},{ }^{2} J_{\mathrm{CF}}=22 \mathrm{~Hz}\right), 130.0\left(\mathrm{~d},{ }^{3} J_{\mathrm{CF}}=9 \mathrm{~Hz}\right), 134.6(\mathrm{~d}$, $\left.{ }^{4} J_{\mathrm{CF}}=4 \mathrm{~Hz}\right), 164.0\left(\mathrm{~d},{ }^{1} J_{\mathrm{CF}}=250 \mathrm{~Hz}\right), 206.6 \mathrm{ppm}$. IR $v$ (neat) $1660 \mathrm{~cm}^{-1}$. Anal. Calcd. for $\mathrm{C}_{24} \mathrm{H}_{22} \mathrm{~F}_{2} \mathrm{O}_{2}$ : C 75.77\%, $\mathrm{H} 5.83 \%$. Found, C $75.81 \%$, H $6.01 \%$.

\section{Synthesis of Poly(ether ketone)s 6, 7, and 10}

To a $10 \mathrm{~mL}$ one-necked flask equipped with a reflux condenser were added difluoride $2 / 4 / 9(0.5 \mathrm{mmol})$, bisphenol $5(0.5 \mathrm{mmol})$, potassium carbonate $(1.5 \mathrm{mmol})$, freshly distilled NMP (1 mL). The NMP solution was stirred at $140^{\circ} \mathrm{C}$ for $1 \mathrm{~h}$ and further stirred at $170^{\circ} \mathrm{C}$ for $7 \mathrm{~h}$. The reaction mixture was poured into methanol and the resulting solid was collected by filtrate suction. The collected solid was washed with water and methanol three times to afford the corresponding poly(ether ketone) 6/7/10.

Poly(ether ketone) 6a: ${ }^{1} \mathrm{H}$ NMR $\delta\left(300 \mathrm{MHz}, \mathrm{CDCl}_{3}\right): 1.25-$ 1.53 (4H, m), $1.71(6 \mathrm{H}, \mathrm{s}), 1.75-1.95(2 \mathrm{H}, \mathrm{m}), 2.01-2.19(2 \mathrm{H}$, $\mathrm{m}), 3.78-3.91(2 \mathrm{H}, \mathrm{m}), 6.80-7.15(8 \mathrm{H}, \mathrm{m}), 7.18-7.30(4 \mathrm{H}, \mathrm{m})$, 7.89-8.08 (4H, m). ${ }^{13} \mathrm{C}$ NMR $\delta\left(75 \mathrm{MHz} \mathrm{CDCl}_{3}\right): 25.9,30.3$, $31.0,42.3,46.9,117.3,119.5,128.2,130.6,130.7,146.7$, 153.4, 161.9, 202.0 ppm. IR v (KBr): $1673 \mathrm{~cm}^{-1}$. Anal. Calcd. for $\mathrm{C}_{35} \mathrm{H}_{32} \mathrm{O}_{4}$ : C $81.37 \%, \mathrm{H} 6.24 \%$. Found, C $81.35 \%, \mathrm{H}$ $6.51 \%$.

Poly(ether ketone) 6b: ${ }^{1} \mathrm{H}$ NMR $\delta\left(300 \mathrm{MHz}, \mathrm{CDCl}_{3}\right)$ : $1.31-$ 1.50 (4H, m), 1.85-1.99 (2H, m), 2.08-2.19 (2H, m), 3.78-3.92 $(2 \mathrm{H}, \mathrm{m}), 6.98-7.11(8 \mathrm{H}, \mathrm{m}), 7.32-7.45(4 \mathrm{H}, \mathrm{m}), 7.97-8.07(4 \mathrm{H}$, m) ppm. ${ }^{13} \mathrm{C} \mathrm{NMR} \delta\left(75 \mathrm{MHz}, \mathrm{CDCl}_{3}\right): 25.9,30.3,47.0,63.8$, $\left(\mathrm{sept},{ }^{2} J_{\mathrm{CF}}=26 \mathrm{~Hz}\right), 118.5,118.9,124.1\left(\mathrm{q},{ }^{1} J_{\mathrm{CF}}=292 \mathrm{~Hz}\right)$, $128.7,130.9,131.6,131.9,156.6,160.5,202.0 \mathrm{ppm}$. IR $v$ (KBr): $1676 \mathrm{~cm}^{-1}$. Anal. Calcd. for $\mathrm{C}_{35} \mathrm{H}_{26} \mathrm{~F}_{6} \mathrm{O}_{4}: \mathrm{C} 67.31 \%, \mathrm{H}$ $4.20 \%$. Found, C $67.07 \%$, H $4.48 \%$.

Poly(ether ketone) 6c: ${ }^{1} \mathrm{H}$ NMR $\delta\left(300 \mathrm{MHz}, \mathrm{CDCl}_{3}\right)$ : 1.19 $1.71(10 \mathrm{H}, \mathrm{m}), 1.73-1.93(2 \mathrm{H}, \mathrm{m}), 1.99-2.18(2 \mathrm{H}, \mathrm{m}), 2.20$ $2.40(4 \mathrm{H}, \mathrm{m}), 3.71-3.88(2 \mathrm{H}, \mathrm{m}), 6.81-7.11(8 \mathrm{H}, \mathrm{m}), 7.14-7.35$ $(4 \mathrm{H}, \mathrm{m}), 7.85-8.09$ (4H, m) ppm. ${ }^{13} \mathrm{C} \mathrm{NMR} \delta(75 \mathrm{MHz}$, $\left.\mathrm{CDCl}_{3}\right): 22.8,25.9,26.1,30.2,37.3,45.5,46.9,117.3,119.6$, $128.6,130.6,130.7,144.6,153.1,161.8,201.9 \mathrm{ppm}$. IR $v$ (KBr): $1673 \mathrm{~cm}^{-1}$. Anal. Calcd. for $\mathrm{C}_{38} \mathrm{H}_{36} \mathrm{O}_{4}: \mathrm{C} 81.99 \%, \mathrm{H}$ $6.52 \%$. Found, C $81.62 \%, \mathrm{H} 6.57 \%$.

Poly(ether ketone) 6d: ${ }^{1} \mathrm{H}$ NMR $\delta\left(300 \mathrm{MHz}, \mathrm{CDCl}_{3}\right): 1.48-$ 1.59 (4H, m), 1.85-1.93 (2H, m), 2.09-2.20 (2H, m), 3.81-3.92 (2H, m), 6.94-7.02 (4H, m), 7.05-7.09 (8H, m), 7.97-8.02 (4H, m) ppm. ${ }^{13} \mathrm{C} \mathrm{NMR} \delta\left(75 \mathrm{MHz}, \mathrm{CDCl}_{3}\right): 25.9,30.2,46.9,116.9$, 120.1, 121.6, 130.6, 130.8, 150.9, 153.9, 162.1, 201.9 ppm. IR v (KBr): $1672 \mathrm{~cm}^{-1}$. Anal. Calcd. for $\mathrm{C}_{32} \mathrm{H}_{26} \mathrm{O}_{5}: \mathrm{C} 78.35 \%, \mathrm{H}$ $5.34 \%$. Found, C $77.90 \%$, H $5.22 \%$.

Poly(ether ketone) 7a: ${ }^{1} \mathrm{H}$ NMR $\delta\left(300 \mathrm{MHz}, \mathrm{CDCl}_{3}\right): 1.50$ $1.79(6 \mathrm{H}, \mathrm{m}), 1.94-2.11(4 \mathrm{H}, \mathrm{m}), 2.22-2.36$ (4H, m), 3.22-3.34 (2H, m), 6.92-7.03 (8H, m), 7.29-7.33 (4H, m), 7.89-7.95 (4H, m) ppm. ${ }^{13} \mathrm{C}$ NMR $\delta\left(75 \mathrm{MHz}, \mathrm{CDCl}_{3}\right): 28.7,31.0,42.3,44.6$, $117.2,119.5,128.3,130.4,130.5,146.8,153.2,162.0$, $201.8 \mathrm{ppm}$. IR $v(\mathrm{KBr}): 1677 \mathrm{~cm}^{-1}$. Anal. Calcd. for $\mathrm{C}_{35} \mathrm{H}_{32} \mathrm{O}_{4}$ : C $81.37 \%$, H $6.24 \%$. Found, C $81.46 \%$, H $6.52 \%$.

Poly(ether ketone) 7b: ${ }^{1} \mathrm{H}$ NMR $\delta\left(300 \mathrm{MHz}, \mathrm{CDCl}_{3}\right): 1.63-$ 1.79 (4H, m), 1.97-2.19 (4H, m), 3.22-3.38 (2H, m), 7.02-7.12 $(8 \mathrm{H}, \mathrm{m}), 7.37-7.44(4 \mathrm{H}, \mathrm{m}), 7.96-8.03(4 \mathrm{H}, \mathrm{m}) \mathrm{ppm} .{ }^{13} \mathrm{C} \mathrm{NMR}$ $\delta\left(75 \mathrm{MHz}, \mathrm{CDCl}_{3}\right): 28.7,44.7,63.8\left(\mathrm{sept},{ }^{2} J_{\mathrm{CF}}=25 \mathrm{~Hz}\right)$, $118.5,118.9,124.1\left(\mathrm{q},{ }^{1} J_{\mathrm{CF}}=286 \mathrm{~Hz}\right), 128.7,130.8,131.5$, $131.9,156.5,160.5,201.5 \mathrm{ppm}$. IR $v(\mathrm{KBr}): 1683 \mathrm{~cm}^{-1}$. Anal. Calcd. for $\mathrm{C}_{35} \mathrm{H}_{26} \mathrm{~F}_{6} \mathrm{O}_{4}$ : C 67.31\%, H 4.20\%. Found, C 67.33\%, H $4.47 \%$. 


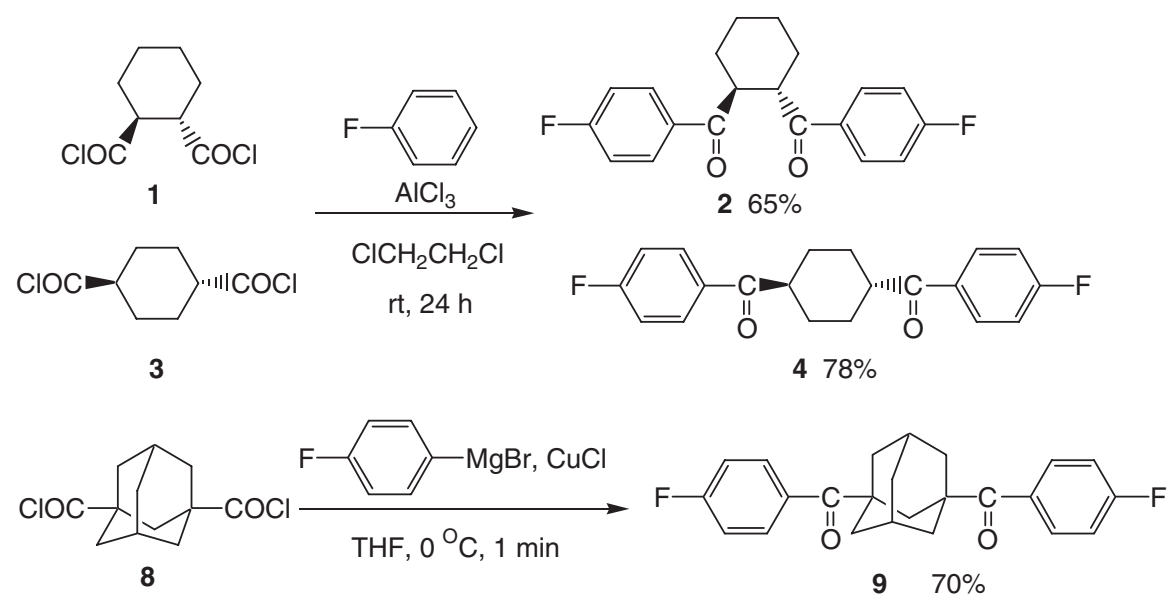

Scheme 1. Preparation of monomers 2, 4, and 9.

Poly(ether ketone) 7c: ${ }^{1} \mathrm{H}$ NMR $\delta\left(300 \mathrm{MHz}, \mathrm{CDCl}_{3}\right): 1.63-$ 1.85 (14H, m), 1.93-2.12 (4H, m), 3.24-3.36 (2H, m), 6.97$7.07(8 \mathrm{H}, \mathrm{m}), 7.27-7.33(4 \mathrm{H}, \mathrm{m}), 7.91-7.99$ (4H, m) ppm. ${ }^{13} \mathrm{C} \mathrm{NMR} \delta\left(75 \mathrm{MHz}, \mathrm{CDCl}_{3}\right): 22.8,26.3,28.7,37.3,44.6$, 45.6, 117.3, 119.6, 119.7, 128.7, 130.5, 144.8, 153.0, 161.9, $201.8 \mathrm{ppm}$. IR $v(\mathrm{KBr}): 1677 \mathrm{~cm}^{-1}$. Anal. Calcd. for $\mathrm{C}_{38} \mathrm{H}_{36} \mathrm{O}_{4}$ : C $81.99 \%$, H $6.52 \%$. Found, C $81.55 \%$, H $6.62 \%$.

Poly(ether ketone) 7d: Insoluble. IR $v(\mathrm{KBr}): 1672 \mathrm{~cm}^{-1}$. Anal. Calcd. for $\mathrm{C}_{32} \mathrm{H}_{26} \mathrm{O}_{5}$ : C 78.35\%, H 5.34\%. Found, C 78.24\%, H $5.43 \%$.

Poly(ether ketone) 10a: ${ }^{1} \mathrm{H}$ NMR $\delta\left(300 \mathrm{MHz}, \mathrm{CDCl}_{3}\right): 1.69$ $(6 \mathrm{H}, \mathrm{s}), 1.77(2 \mathrm{H}, \mathrm{s}), 1.95-2.13(8 \mathrm{H}, \mathrm{m}), 2.23-2.34(4 \mathrm{H}, \mathrm{m})$, $6.95(8 \mathrm{H}, \mathrm{d}, J=8.7 \mathrm{~Hz}), 7.24(4 \mathrm{H}, \mathrm{d}, J=8.7 \mathrm{~Hz}), 7.70(4 \mathrm{H}, \mathrm{d}$, $J=8.7 \mathrm{~Hz}) \mathrm{ppm} .{ }^{13} \mathrm{C} \mathrm{NMR} \delta\left(75 \mathrm{MHz}, \mathrm{CDCl}_{3}\right): 28.3,31.0$, 35.5, 38.6, 40.7, 42.3, 47.2, 117.0, 119.4, 128.2, 130.2, 132.5, 146.5, 153.5, 160.1, 206.2 ppm. IR $v(\mathrm{KBr}): 1663 \mathrm{~cm}^{-1}$. Anal. Calcd. for $\mathrm{C}_{39} \mathrm{H}_{36} \mathrm{O}_{4}$ : C $82.37 \%$, H 6.38\%. Found, C $82.44 \%, \mathrm{H}$ $6.57 \%$.

Poly(ether ketone) 10b: ${ }^{1} \mathrm{H}$ NMR $\delta\left(300 \mathrm{MHz}, \mathrm{CDCl}_{3}\right): 1.78$ (2H, s), 1.95-2.15 (8H, m), $2.30(4 \mathrm{H}, \mathrm{s}), 6.92-7.13(8 \mathrm{H}, \mathrm{m})$, $7.39(4 \mathrm{H}, \mathrm{d}, J=8.1 \mathrm{~Hz}), 7.72(4 \mathrm{H}, \mathrm{d}, J=8.1 \mathrm{~Hz}) \mathrm{ppm}$. ${ }^{13} \mathrm{C} \mathrm{NMR} \delta\left(75 \mathrm{MHz}, \mathrm{CDCl}_{3}\right): 28.2,35.5,38.4,40.5,47.2,64.2$ (sept, $\left.{ }^{2} J_{\mathrm{CF}}=30 \mathrm{~Hz}\right), 118.2,118.6,123.8(\mathrm{~m}), 128.5,130.1$, 131.9, 133.7, 156.8, 158.5, $206.5 \mathrm{ppm}$. IR $v(\mathrm{KBr}): 1667 \mathrm{~cm}^{-1}$. Anal. Calcd. for $\mathrm{C}_{39} \mathrm{H}_{30} \mathrm{~F}_{6} \mathrm{O}_{4}: \mathrm{C} 69.23 \%$, $\mathrm{H} 4.47 \%$. Found, C $69.41 \%, \mathrm{H} 4.51 \%$.

Poly(ether ketone) 10c: ${ }^{1} \mathrm{H}$ NMR $\delta\left(300 \mathrm{MHz}, \mathrm{CDCl}_{3}\right): 1.45-$ $1.61(10 \mathrm{H}, \mathrm{m}), 1.77(2 \mathrm{H}, \mathrm{s}), 1.95-2.15(8 \mathrm{H}, \mathrm{m}), 2.21-2.34(4 \mathrm{H}$, m), 6.89-6.99 (8H, m), 7.22-7.30 (4H, m), $7.70(4 \mathrm{H}, \mathrm{d}$, $J=8.7 \mathrm{~Hz}) \mathrm{ppm} .{ }^{13} \mathrm{C} \mathrm{NMR} \delta\left(75 \mathrm{MHz}, \mathrm{CDCl}_{3}\right): 22.8,26.3$, 28.4, 35.5, 37.3, 38.6, 40.7, 45.6, 47.3, 117.1, 119.5, 128.6, $130.2,132.5,144.5,153.3,160.1,206.3 \mathrm{ppm}$. IR $v(\mathrm{KBr})$ : $1664 \mathrm{~cm}^{-1}$. Anal. Calcd. for $\mathrm{C}_{42} \mathrm{H}_{40} \mathrm{O}_{4}$ : C $82.86 \%, \mathrm{H} 6.62 \%$. Found, C $82.96 \%$, H $6.76 \%$.

Poly(ether ketone) 10d: ${ }^{1} \mathrm{H}$ NMR $\delta\left(300 \mathrm{MHz}, \mathrm{CDCl}_{3}\right): 1.78$ $(2 \mathrm{H}, \mathrm{s}), 1.95-2.15(8 \mathrm{H}, \mathrm{m}), 2.31(4 \mathrm{H}, \mathrm{s}), 6.95(4 \mathrm{H}, \mathrm{d}, J=$ $8.7 \mathrm{~Hz}), 7.00-7.08(8 \mathrm{H}, \mathrm{m}), 7.72(4 \mathrm{H}, \mathrm{d}, J=8.7 \mathrm{~Hz}) \mathrm{ppm}$. ${ }^{13} \mathrm{C} \mathrm{NMR} \delta\left(75 \mathrm{MHz}, \mathrm{CDCl}_{3}\right): 28.3,35.5,38.6,40.7,47.2$, $116.7,120.1,121.5,130.2,132.5,151.1,153.8,160.4,206.3$ ppm. IR $v(\mathrm{KBr}): 1662 \mathrm{~cm}^{-1}$. Anal. Calcd. for $\mathrm{C}_{36} \mathrm{H}_{30} \mathrm{O}_{5}: \mathrm{C}$ $79.68 \%$, H 5.57\%. Found, C 79.59\%, H 5.80\%.

\section{RESULTS AND DISCUSSION}

\section{Synthesis of Poly(ether ketone)s 6 and 7}

Two isomeric bis(4-fluorobenzoyl)cyclohexanes $\mathbf{2}$ and $\mathbf{4}$ were prepared through $\mathrm{AlCl}_{3}$-mediated regioselective FriedelCrafts acylation reaction of cyclohexanedicarbonyl dichlorides $\mathbf{1}$ and $\mathbf{3}$ with fluorobenzene in 1,2-dichloroethane. Nucleophilic aromatic substitution polymerization of difluoride monomer 2 with bisphenol A (5a) was performed in the presence of $\mathrm{K}_{2} \mathrm{CO}_{3}$ in NMP. Low-molecular-weight poly(ether ketone) (6a) was obtained when the reaction mixture was stirred at $170{ }^{\circ} \mathrm{C}$ at $8 \mathrm{~h}$ (Run 1). On the other hand, high-molecular-weight poly(ether ketone) (6a) was obtained when the reaction mixture was stirred at $140^{\circ} \mathrm{C}$ at $1 \mathrm{~h}$ and then $170^{\circ} \mathrm{C}$ at $7 \mathrm{~h}$ (Run 2). Probably, potassium diphenoxide is formed at $140{ }^{\circ} \mathrm{C}$ and reacts with difluoride monomer 2 at $170^{\circ} \mathrm{C}$ to afford the corresponding poly(ether ketone).

The results of polymerization of difluoride $\mathbf{2}$ with bisphenols 5a-d are shown in Table I.

The resulting poly(ether ketone)s (6a-d) were high-molecular-weight polymers on the basis of GPC measurements. In addition, poly(ether ketone)s 6a-d have excellent solubility in organic solvents such as $\mathrm{CHCl}_{3}$ and THF (Table IV). Introduc-

Table I. Polymerization of trans-1,2-bis(4-fluorobenzoyl)cyclohexane (2) with bisphenols 5a-d

\begin{tabular}{cccccc}
\hline Run & Bisphenol & Yield $/ \%$ & $M_{\mathrm{n}}{ }^{\mathrm{a})}$ & $T_{\mathrm{g}} /{ }^{\circ} \mathrm{C}^{\mathrm{b})}$ & $T_{\mathrm{d} 5} /{ }^{\circ} \mathrm{C}^{\mathrm{c})}$ \\
\hline 1 & $\mathbf{5 a}^{\mathrm{d})}$ & 65 & 3000 & - & - \\
2 & $\mathbf{5 a}$ & $93(6 \mathbf{a})$ & 18000 & 187 & 437 \\
3 & $\mathbf{5 b}$ & $97(6 \mathbf{b})$ & 20000 & 197 & 462 \\
4 & $\mathbf{5 c}$ & $92(6 \mathbf{c})$ & 15000 & 199 & 454 \\
5 & $\mathbf{5 d}$ & $100(6 \mathbf{d})$ & 12000 & 187 & 438 \\
\hline
\end{tabular}

Reaction conditions: difluoride 2, $0.5 \mathrm{mmol}$; bisphenol $5,0.5 \mathrm{mmol}$; potassium carbonate, $1.6 \mathrm{mmol}$; NMP, $1 \mathrm{~mL} ; 140^{\circ} \mathrm{C}, 1 \mathrm{~h}$ then $170^{\circ} \mathrm{C}, 7 \mathrm{~h}$. a) Estimated by GPC (eluent; $\mathrm{CHCl}_{3}$ ) based on polystyrene standards. b) Determined on the basis of DSC curves. Heating rate: $10 \mathrm{~K} / \mathrm{min}$. c) Temperature where a $5 \%$ weight loss occurs. Heating rate: $10 \mathrm{~K} / \mathrm{min}$ d) The reaction was performed at $170^{\circ} \mathrm{C}$ for $8 \mathrm{~h}$. 
Table II. Polymerization of trans-1,4-bis(4-fluorobenzoyl)cyclohexane (4) with bisphenols 5 a-d

\begin{tabular}{cccccc}
\hline Run & Bisphenol & Yield $/ \%$ & $M_{\mathrm{n}}{ }^{\mathrm{a})}$ & $T_{\mathrm{g}} /{ }^{\circ} \mathrm{C}^{\mathrm{b})}$ & $\left.T_{\mathrm{d} 5} /{ }^{\circ} \mathrm{C}^{\mathrm{c}}\right)$ \\
\hline 1 & 5a & 89(7a) & 19000 & 176 & 432 \\
2 & 5b & $99(7 \mathbf{b})$ & 14000 & 175 & 458 \\
3 & 5c & 87(7c) & 18000 & 187 & 453 \\
4 & 5d & 93(7d) & insoluble & 171 & 456 \\
\hline
\end{tabular}

Reaction conditions: difluoride 4, $0.5 \mathrm{mmol}$; bisphenol $5,0.5 \mathrm{mmol}$; potassium carbonate, $1.6 \mathrm{mmol}$; NMP, $1 \mathrm{~mL} ; 140^{\circ} \mathrm{C}, 1 \mathrm{~h}$ then $170^{\circ} \mathrm{C}, 7 \mathrm{~h}$.

a) Estimated by GPC (eluent; $\mathrm{CHCl}_{3}$ ) based on polystyrene standards.

b) Determined on the basis of DSC curves. Heating rate: $10 \mathrm{~K} / \mathrm{min}$.

c) Temperature where a $5 \%$ weight loss occurs. Heating rate: $10 \mathrm{~K} / \mathrm{min}$.
Table III. Polymerization of 1,3-bis(4-fluorobenzoyl)adamantane (9) with bisphenols 5a-d

\begin{tabular}{cccrcc}
\hline Run & Bisphenol & Yield $/ \%$ & \multicolumn{1}{c}{$M_{\mathrm{n}}{ }^{\mathrm{a})}$} & $T_{\mathrm{g}} /{ }^{\circ} \mathrm{C}^{\mathrm{b})}$ & $\left.T_{\mathrm{d} 5} /{ }^{\circ} \mathrm{C}^{\mathrm{c}}\right)$ \\
\hline 1 & 5a & $99(\mathbf{1 0 a})$ & 35000 & 146 & 490 \\
2 & 5b & $67(\mathbf{1 0 b})$ & 8000 & 146 & 500 \\
3 & 5c & $98(\mathbf{1 0 c})$ & 13000 & 154 & 492 \\
4 & 5d & 99(10d) & 28000 & 140 & 489 \\
\hline
\end{tabular}

Reaction conditions: difluoride 9, $0.5 \mathrm{mmol}$; bisphenol $5,0.5 \mathrm{mmol}$; potassium carbonate, $1.6 \mathrm{mmol}$; NMP, $1 \mathrm{~mL} ; 140^{\circ} \mathrm{C}, 1 \mathrm{~h}$ then $170^{\circ} \mathrm{C}, 7 \mathrm{~h}$

a) Estimated by GPC (eluent; $\mathrm{CHCl}_{3}$ ) based on polystyrene standards

b) Determined on the basis of DSC curves. Heating rate: $10 \mathrm{~K} / \mathrm{min}$.

c) Temperature where a $5 \%$ weight loss occurs. Heating rate: $10 \mathrm{~K} / \mathrm{min}$.

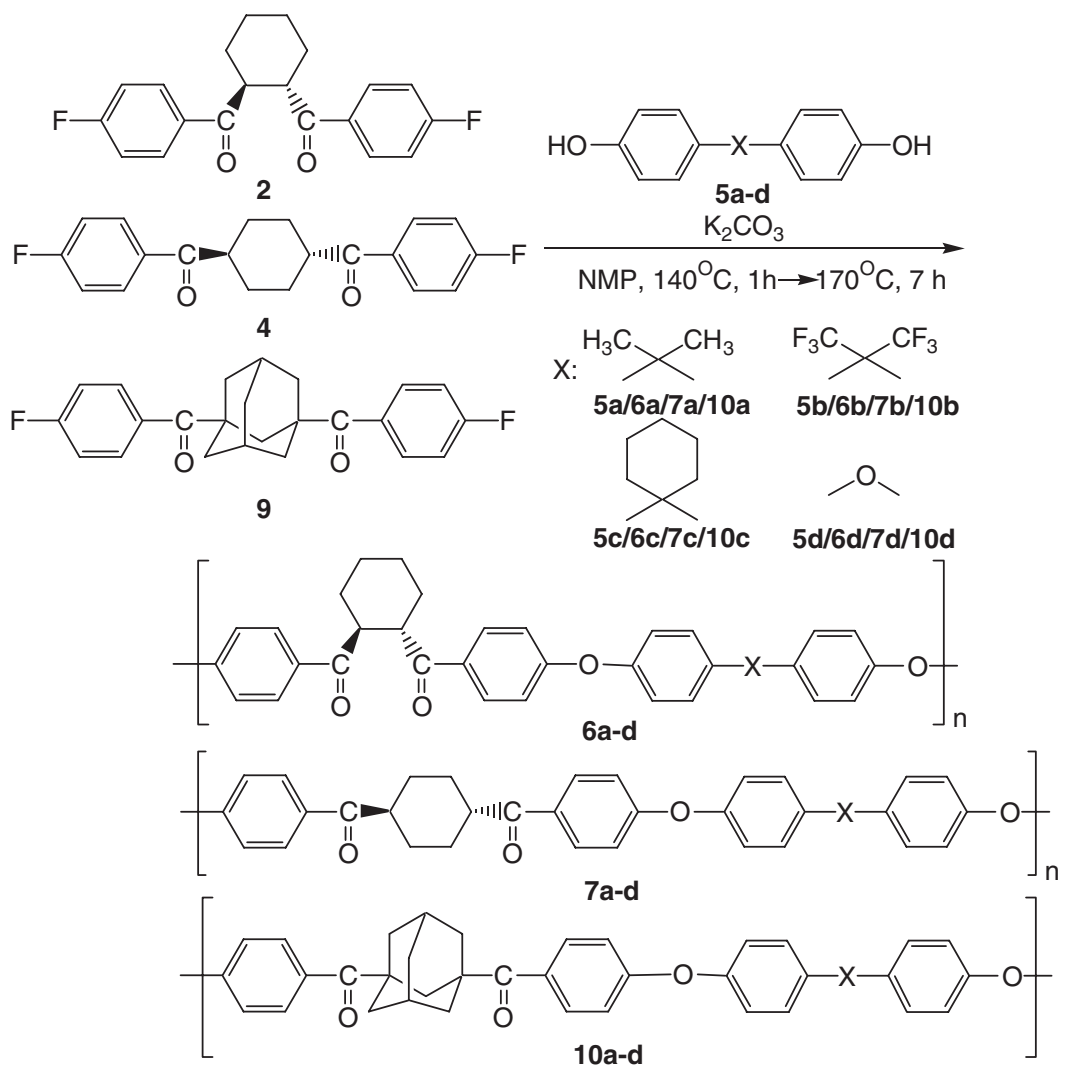

Scheme 2. Synthesis of aromatic poly(ether ketone)s 6, 7, and 10.

tion of trans-1,2-cyclohexanediyl units to poly(ether ketone) backbones might improve the solubility in organic solvents.

Similarily, nucleophilic aromatic substitution polymerization of regioisomeric difluoride monomer 4 with bisphenols $\mathbf{5 a -}$ d was carried out. The results are shown in Table II. Although poly(ether ketone)s 7a-c are soluble to organic solvents, polyketone 7d, which is derived from 4,4'-oxybiphenol, is insoluble to $\mathrm{CHCl}_{3}$. Glass transition temperatures $\left(T_{\mathrm{g}}\right.$ 's) of poly(ether ketone)s 7a-d are as high as those of polyketones 6a-d. Temperatures where $5 \%$ weight losses occur $\left(T_{\mathrm{d} 5}\right)$ for poly(ether ketone)s 7a-d are also as high as those of poly(ether ketone)s 6a-d.

\section{Synthesis of Adamantanediyl-containing Polyketones 10}

Friedel-Crafts acylation reaction of fluorobenzene with 1,3adamantanedicarbonyl dichloride (8) was attempted to obtain the corresponding difluoride (9). However, complex mixtures including difluoride $\mathbf{9}$ as the minor product were obtained due to bulkiness of adamantane units (Scheme 2). Difluoride 9 was prepared via the reaction of dicarbonyl dichloride 8 with 4fluorophenylmagnesium bromide in the presence of $\mathrm{CuCl}$ (Scheme 2). ${ }^{13}$

Nucleophilic aromatic substitution polymerization of difluoride monomer 9 with bisphenols 5a-d was carried out. The results are shown in Table III. Poly(ether ketone)s 10a, 10c, and 10d were obtained as high-molecular-weight polymers in good yields. On the other hand, poly(ether ketone) 10b, which is derived from $\mathrm{CF}_{3}$-disubstituted bisphenol $(\mathbf{5 b})$, was obtained as a lower-molecular-weight polymer $\left(M_{\mathrm{n}} 8000\right)$ in lower yield. The reason might be due to both low reactivity of bisphenol $\mathbf{5 b}$, based on electron withdrawing effect by $\mathrm{CF}_{3}$ groups, and bulkiness of 1,3-adamantanediyl units. 
Table IV. Solubility of poly(ether ketone)s $\mathbf{6 , 7}$, and 10

\begin{tabular}{|c|c|c|c|c|c|c|c|}
\hline Polymer & $m$-cresol & THF & $\mathrm{CHCl}_{3}$ & DMSO & DMF & NMP & $\mathrm{H}_{2} \mathrm{SO}_{4}$ \\
\hline $6 a$ & ++ & ++ & ++ & \pm & \pm & ++ & ++ \\
\hline $6 b$ & + & ++ & ++ & \pm & ++ & ++ & ++ \\
\hline $6 c$ & ++ & ++ & ++ & \pm & \pm & ++ & ++ \\
\hline $6 d$ & ++ & ++ & ++ & \pm & ++ & ++ & ++ \\
\hline $7 a$ & ++ & ++ & ++ & \pm & \pm & ++ & ++ \\
\hline $7 b$ & \pm & ++ & ++ & \pm & \pm & ++ & ++ \\
\hline 7c & \pm & ++ & ++ & \pm & \pm & ++ & ++ \\
\hline $7 d$ & - & \pm & - & - & \pm & \pm & ++ \\
\hline $10 a$ & ++ & ++ & ++ & - & \pm & ++ & ++ \\
\hline $10 \mathrm{~b}$ & ++ & ++ & ++ & - & \pm & ++ & ++ \\
\hline $10 c$ & ++ & ++ & ++ & - & \pm & ++ & ++ \\
\hline $10 d$ & ++ & ++ & ++ & - & \pm & ++ & ++ \\
\hline
\end{tabular}

Solubility: $(++)$ : soluble at room temperature. $(+)$ : soluble upon heating. $( \pm)$ : partially soluble or swelling. $(-)$ : insoluble.
We have reported that $T_{\mathrm{g}}$ 's of the polyesters containing 1,3adamantanediyl units are $184-222^{\circ} \mathrm{C}$ and higher than those of the polyesters containing 1,2-cyclohexanediyl units (110$\left.156^{\circ} \mathrm{C}\right) .{ }^{12}$ On the other hand, $T_{\mathrm{g}}$ 's of poly(ether ketone)s 10 (Ad) are $140-154^{\circ} \mathrm{C}$ and lower than those of poly(ether ketone)s 6 (1,2-Cy) and 7 (1,4-Cy) as shown in Table III. There is a possibility that the bent structures of 1,3-adamantanediyl units give flexibility to the main chains of poly(ether ketone)s 10. This might decrease $T_{\mathrm{g}}$ 's of poly(ether ketone)s 10. On the contrary, stronger interaction between ester groups might suppress the rotation based on the bent structures of 1,3adamantanediyl units in the main chains of the polyesters, which increases $T_{\mathrm{g}}$ 's of the polyesters containing 1,3-adamantanediyl units.

Figure 1 shows TG thermograms of poly(ether ketone)s $\mathbf{6 a}(1,2-\mathrm{Cy}), \mathbf{7 a}(1,4-\mathrm{Cy})$, and $\mathbf{1 0 a}(\mathrm{Ad})$. Temperatures where $5 \%$ weight losses occur $\left(T_{\mathrm{d} 5}\right)$ for poly(ether ketone)s 10a-d are in the region of $489-500^{\circ} \mathrm{C}$. $T_{\mathrm{d} 5}$ 's of poly(ether ketone)s $\mathbf{1 0}(\mathrm{Ad})$

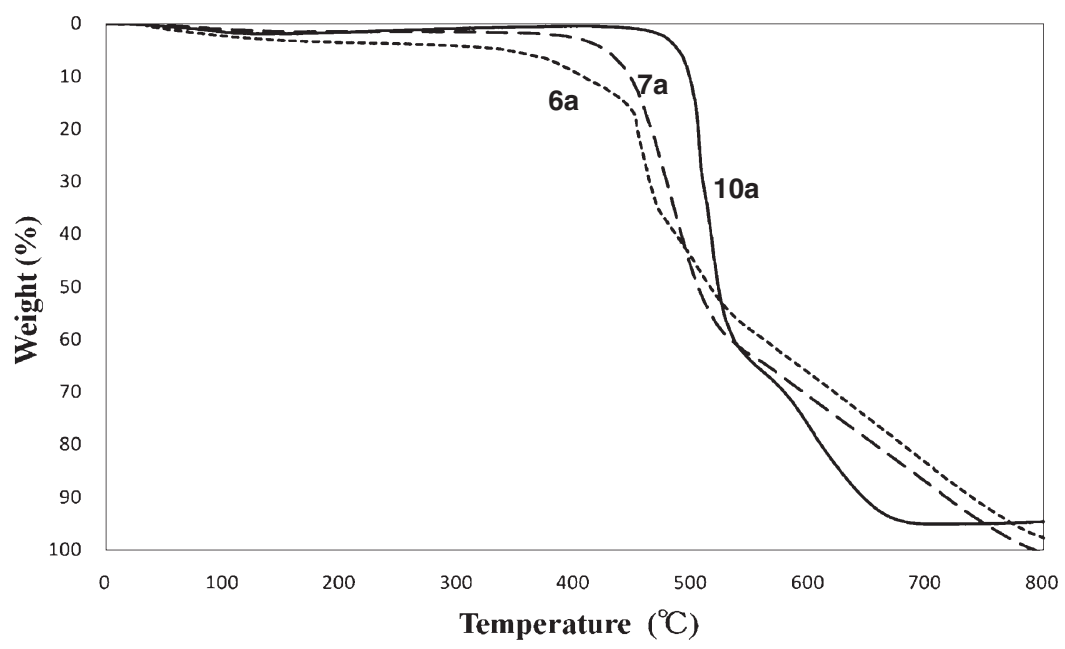

Figure 1. TG thermograms of poly(ether ketone)s $6 a, 7 a$, and $10 a$

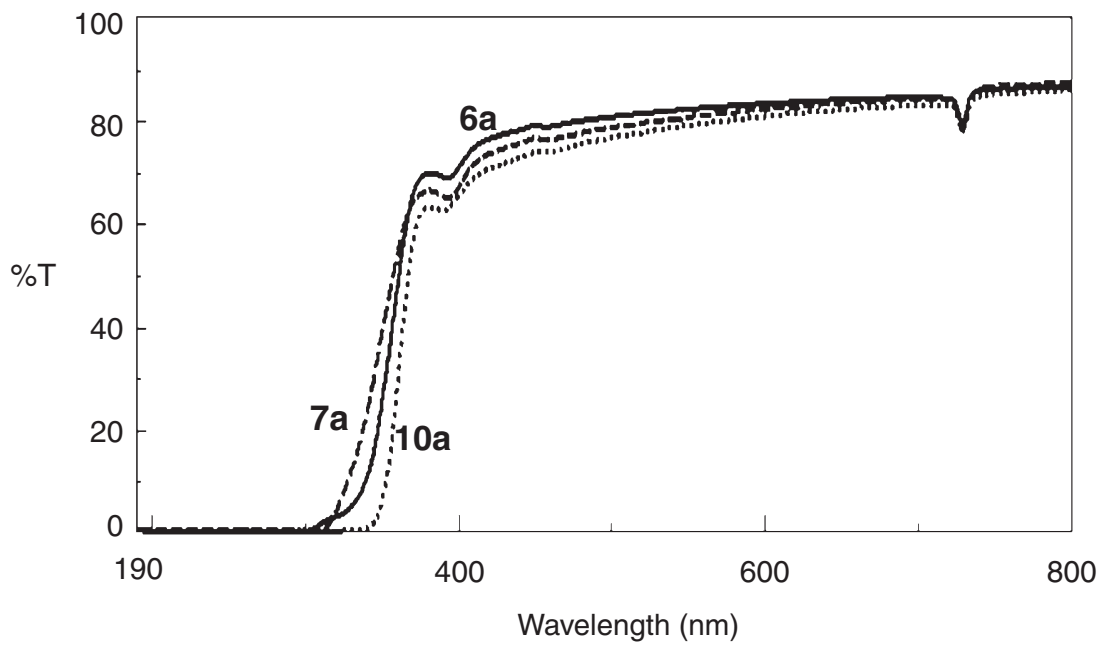

Figure 2. UV-vis spectra of poly(ether ketone)s $6 \mathbf{a}, 7 \mathbf{a}$, and $\mathbf{1 0 a}$. 
are higher than those of poly(ether ketone)s $\mathbf{6}(1,2-\mathrm{Cy})$ and 7(1,4-Cy), probably due to bulkiness of adamantane units.

UV spectra of poly(ether ketone) films $\mathbf{6 a}(1,2-\mathrm{Cy}), \mathbf{7} \mathbf{a}(1,4-$ $\mathrm{Cy}$ ), and $\mathbf{1 0 a}(\mathrm{Ad})$, which are prepared by casting from $\mathrm{CHCl}_{3}$, are shown in Figure 2. The spectra indicate that the films are transparent in wavelength above $310 \mathrm{~nm}$.

\section{CONCLUSIONS}

Aromatic poly(ether ketone)s 6, 7, and $\mathbf{1 0}$ containing cyclohexanediyl and adamantanediyl units were synthesized through nucleophilic aromatic substitution polymerization of alicyclic difluorides 2, 4, and 9 with bisphenols 5a-d in the presence of $\mathrm{AlCl}_{3}$ in NMP. Most of poly(ether ketone)s 6, 7, and $\mathbf{1 0}$ have excellent thermal stability, solubility in organic solvents, and transparency.

Acknowledgment. This work is supported by the Ministry of Education, Culture, Sports, Science, and Technology of Japan.

Received: April 15, 2008

Accepted: June 9, 2008

Published: July 24, 2008

\section{REFERENCES}

1. "Comprehensive Polymer Science," P. A. Staniland, in G. C. Eastmond, A. Ledwith, S. Russo, and P. Sigwalt, Ed., Pergamon Press, Oxford, UK 1989, vol. 5, p 483.

2. K. Maeyama, Kino Zairyo, 27, 47 (2007).

3. K. Maeyama, I. Hikiji, K. Ogura, A. Okamoto, K. Ogino, H. Saito, and N. Yonezawa, Polym. J., 37, 736 (2005).

4. K. Maeyama, S. Maeda, K. Ogino, H. Saito, and N. Yonezawa, React. Funct. Polym., 65, 229 (2005).

5. K. Maeyama, K. Ogura, A. Okamoto, K. Ogino, H. Saito, and N. Yonezawa, Polym. J., 37, 736 (2005).

6. T. Matumoto, J. Synth. Org. Chem. Jpn., 58, 776 (2000).

7. Q. Jin, T. Yamashita, K. Horie, R. Yokota, and I. Mita, J. Polym. Sci., Part A: Polym. Chem., 31, 2345 (1993).

8. H. Seino, A. Mochizuki, and M. Ueda, J. Polym. Sci., Part A: Polym. Chem., 37, 3584 (1999).

9. K. Fukukawa, Y. Shibasaki, and M. Ueda, Macromolecules, 37, 8256 (2004).

10. K. Obuchi, M. Komatsu, and K. Minami, Proceedings of APIE-The International Society for Optical Engineering, 6671 (2001).

11. N. Nishimura, K. Maeyama, and A. Toyota, Progress in Olefin Polymerization Catalysts and Polyolefin Materials, 193 (2006).

12. K. Maeyama, K. Akiba, and H. Saito, Polym. J., 40, 629 (2008).

13. R. Vicha and M. Potacek, Tetrahedron, 61, 83 (2005). 